Bull. Austral. Math. Soc.

VoL. 44 (1991) [63-69]

\title{
COMMUTATIVITY OF RINGS SATISFYING CERTAIN POLYNOMIAL IDENTITIES
}

\author{
Hazar Abu-Khuzam, Howard Bell and Adil Yaqub
}

\begin{abstract}
It is shown that an $n$-torsion-free ring $R$ with identity such that, for all $x, y$ in $R$, $x^{n} y^{n}=y^{n} x^{n}$ and $(x y)^{n+1}-x^{n+1} y^{n+1}$ is central, must be commutative. It is also shown that a periodic $n$-torsion-free ring (not necessarily with identity) for which $(x y)^{n}-(y x)^{n}$ is always in the centre is commutative provided that the nilpotents of $R$ form a commutative set. Further, examples are given which show that all the hypotheses of both theorems are essential.
\end{abstract}

$R$ is called periodic if for every $x$ in $R$, there exist distinct positive integers $m=$ $m(x), n=n(x)$ such that $x^{m}=x^{n}$. By a theorem of Chacron (see [6, Theorem 1]), $R$ is periodic if and only if for each $x \in R$, there exists a positive integer $k=k(x)$ and a polynomial $f(\lambda)=f_{x}(\lambda)$ with integer coefficients such that $x^{k}=x^{k+1} f(x)$.

Throughout, $R$ is an associative ring, $N$ denotes the set of nilpotent elements of $R, Z$ denotes the centre of $R, C(R)$ denotes the commutator ideal of $R$, and $[x, y]$ denotes the commutator $x y-y x$. We start with our first theorem:

THEOREM 1. Let $R$ be a ring with identity and let $n$ be a fixed positive integer. Suppose that $R$ is $n$-torsion-free, and that for all $x, y$ in $R, x^{n} y^{n}=y^{n} x^{n}$ and $(x y)^{n+1}-$ $x^{n+1} y^{n+1}$ is in the centre $Z$ of $R$. Then $R$ is commutative.

In preparation for the proof of Theorem 1, we state the following known lemmas $[2,11,5]$.

LEMMA 1. If $[x, y]$ commutes with $x$, then $\left[x^{k}, y\right]=k x^{k-1}[x, y]$ for all positive integers $k$.

LEMMA 2. Suppose that $R$ is a ring with identity 1 . If $x^{m}[x, y]=0$ and $(x+1)^{m}[x, y]=0$ for some $x, y$ in $R$ and some integer $m>0$, then $[x, y]=0$. $A$ similar statement holds if we assume $[x, y] x^{m}=0$ and $[x, y](x+1)^{m}=0$ instead.

LеммA 3. Let $R$ be an $n$-torsion-free ring with identity 1 such that $\left[x^{n}, y^{n}\right]=0$ for all $x, y$ in $R$. Let $N$ denote the set of nilpotent elements of $R$. Then

(i) $a \in N, x \in R$ imply $\left[a, x^{n}\right]=0$.

(ii) $a \in N, b \in N$ imply $[a, b]=0$.

Received 6th August, 1990.

Copyright Clearance Centre, Inc. Serial-fee code: 0004-9729/91 SA2.00+0.00. 
Proof of Theorem 1: By hypothesis, $\left[x^{n}, y^{n}\right]=0$ for all $x, y$ in $R$ and hence, by [8], the commutator ideal is nil. This implies that the set of nilpotent elements $N$ forms an ideal. Hence, by Lemma 3 (ii), $N$ is a commutative ideal. This implies that

$$
N^{2} \subseteq Z
$$

Let $a \in N, b \in R$. Then by hypothesis,

$$
\begin{aligned}
& ((a+1) b)^{n+1}-(a+1)^{n+1} b^{n+1} \in Z, \text { and } \\
& (b(a+1))^{n+1}-b^{n+1}(a+1)^{n+1} \in Z .
\end{aligned}
$$

Subtracting (3) from (2), and using the fact that $N^{2} \subseteq Z$ we get

$$
a b^{n+1}-b^{n+1} a-(n+1) a b^{n+1}+(n+1) b^{n+1} a \in Z,
$$

and thus $n\left[a, b^{n+1}\right] \in Z$. Hence, since $R$ is $n$-torsion-free, we get

$$
\left[a, b^{n+1}\right] \in Z,(a \in N, b \in R)
$$

Therefore,

$$
\left[a, b^{n+1}\right]=[a, b] b^{n}+b\left[a, b^{n}\right] \in Z, \text { by }(4) .
$$

But, by Lemma 3(i), $\left[a, b^{n}\right]=0$, and hence by (5),

$$
[a, b] b^{n} \in Z,(a \in N, b \in R) .
$$

Thus,

$$
\left[[a, b] b^{n}, b\right]=0=[[a, b], b] b^{n} \text {. }
$$

Replacing $b$ by $b+1$ in the above argument and using Lemma 2, we see that

$$
[[a, b], b]=0,(a \in N, b \in R) .
$$

Using Lemma 3(i), (7), and Lemma 1 we get

$$
0=\left[a, b^{n}\right]=n b^{n-1}[a, b] .
$$

Since $R$ is $n$-torsion-free, we conclude that $b^{n-1}[a, b]=0$. Putting $b+1$ instead of $b$, and using Lemma 2, we get

$$
[a, b]=0,(a \in N, b \in R)
$$


Thus, the nilpotent elements are central and hence (since $C(R)$ is nil)

$$
[x, y] \in Z, \text { for all } x, y \text { in } R \text {. }
$$

Using (8) and Lemma 1 , we have $0=\left[x^{n}, y^{n}\right]=n x^{n-1}\left[x, y^{n}\right]$. Now, using the fact that $R$ is $n$-torsion-free and Lemma 2 we get $\left[x, y^{n}\right]=0$ for all $x, y$ in $R$. Similarly, $0=\left[x, y^{n}\right]=n y^{n-1}[x, y]$ yields $[x, y]=0$ for all $x, y$ in $R$. This completes the proof of Theorem 1 .

In preparation for the proof of the next theorem, we state the following lemma which is proved in [4].

Lemma 4. Let $R$ be a periodic ring such that $N$ is commutative. Then the commutator ideal of $R$ is nil, and $N$ forms an ideal of $R$.

THEOREM 2. Let $n$ be a fixed positive integer and let $R$ be an $n$-torsion-free periodic ring (not necessarily with identity) such that $(x y)^{n}-(y x)^{n} \in Z$. If $N$ is commutative, then $R$ is commutative.

Proof: Consider first the case that $R$ has an identity 1. By Lemma 4, $N$ is an ideal of $R$. Also, since $N$ is commutative,

$$
N^{2} \subseteq Z
$$

Let $a \in N, b \in R$. Taking $x=(1+a) b, y=(1+a)^{-1}$, the hypothesis $(x y)^{n}-(y x)^{n} \in$ $Z$ yields

$$
(1+a) b^{n}(1+a)^{-1}-b^{n} \in Z,
$$

and hence

$$
\left[(1+a) b^{n}(1+a)^{-1}-b^{n}\right](1+a)=(1+a)\left[(1+a) b^{n}(1+a)^{-1}-b^{n}\right] .
$$

Therefore

$$
\begin{gathered}
(1+a) b^{n}-b^{n}(1+a)=(1+a)\left[(1+a) b^{n}(1+a)^{-1}-b^{n}\right], \\
a b^{n}-b^{n} a=(1+a)\left[(1+a) b^{n}(1+a)^{-1}-b^{n}\right] .
\end{gathered}
$$

Since $N$ is a commutative ideal, $(1+a)\left(a b^{n}-b^{n} a\right)=a b^{n}-b^{n} a$, and hence by (10),

$$
(1+a)\left(a b^{n}-b^{n} a\right)=(1+a)\left[(1+a) b^{n}(1+a)^{-1}-b^{n}\right] .
$$

Further, since $a \in N, 1+a$ is a unit in $R$, and thus

$$
a b^{n}-b^{n} a=(1+a) b^{n}(1+a)^{-1}-b^{n} \in Z \text {, by (9). }
$$

Thus, 


$$
\left[a, b^{n}\right] \in Z,(a \in N, b \in R)
$$

Now, suppose $x_{1}, \ldots, x_{k} \in R$. Since $R / C(R)$ is commutative,

$$
\left(x_{1} \ldots x_{k}\right)^{n}-x_{1}^{n} \ldots x_{k}^{n} \in C(R) \subseteq N, \text { by Lemma } 4
$$

But $N$ is commutative, and hence

$$
\left[a,\left(x_{1} \ldots x_{k}\right)^{n}\right]=\left[a, x_{1}^{n} \ldots x_{k}^{n}\right],(a \in N)
$$

Combining (11) and (12), we conclude that

$$
\left[a, x_{1}^{n} \ldots x_{k}^{n}\right] \in Z,\left(a \in N ; x_{1}, \ldots, x_{k} \in R ; \text { any } k \geqslant 1\right)
$$

Let $S$ be the subring of $R$ generated by the $n$-th powers of elements of $R$. Then, by (13),

$$
[a, x] \in Z(S) \text { fór all } a \in N(S), x \in S,
$$

(here $Z(S)$ and $N(S)$ denote the centre of $S$ and the set of nilpotents of $S$, respectively). Combining the facts that $S$ is periodic, $N(S)$ is commutative, and (14), a theorem of [3] shows that $S$ is commutative, and hence

$$
\left[x^{n}, y^{n}\right]=0 \text { for all } x, y \in R
$$

Note that $R$ is an $n$-torsion-free ring with identity satisfying (15) and the hypothesis " $(x y)^{n}-(y x)^{n}$ is always central", and hence by Theorem 1 of [1], $R$ is commutative (in the event $R$ happens to have an identity).

We now consider the general case. We begin with the following two claims.

Claim 1. The idempotents of $R$ are central.

Let $e^{2}=e \in R, r \in R$. By hypothesis,

$$
[e(e+e r-e r e)]^{n}-[(e+e r-e r e) e]^{n} \in Z,
$$

and hence $e r-e r e \in Z$. Therefore,

$$
e r-e r e=e(e r-e r e)=(e r-e r e) e=0,
$$

and thus $e r=e r e$. Similarly, $r e=e r e$, and the claim follows. 
Claim 2. If $\sigma: R \rightarrow S$ is a homomorphism of $R$ onto $S$, then the nilpotents of $S$ coincide with $\sigma(N)$, where $N$ is the set of nilpotents of $R$.

This claim was essentially proved in [9].

To complete the proof of the theorem, first recall that $R$ is isomorphic to a subdirect sum of subdirectly irreducible rings $R_{i}(i \in \Gamma)$. Suppose that

$$
\sigma_{i}: R \rightarrow R_{i}
$$

is the natural homomorphism of $R$ onto $R_{i}$. Let $x_{i} \in R_{i}$ and let $\sigma_{i}(x)=x_{i}, x \in R$. Since $R$ is periodic,

$$
x^{*}=x^{r} \text { for some integers } s>r>0
$$

and hence

$$
e=x^{(\bullet-r) r} \text { is idempotent. }
$$

By Claim $1, e$ is central in $R$, and hence $\sigma_{i}(e)$ is a central idempotent of $R_{i}$. Since $R_{i}$ is subdirectly irreducible, $\sigma_{i}(e)=0$ or $\sigma_{i}(e)=1_{i}$ (if $1_{i} \in R_{i}$ ).

CASE $1 . R_{i}$ does not have an identity.

In this case, $\sigma_{i}(e)=0$ and hence (see (16)), $x_{i}^{(s-r) r}=0$. Thus $R_{i}$ is nil and hence, by Claim 2,

$$
R_{i}=\sigma_{i}(N) \text {. }
$$

By hypothesis, $N$ is commutative; therefore $R_{i}$ is commutative.

CASE 2. $R_{i}$ has an identity $1_{i}$.

Note that $R_{i}$ need not be $n$-torsion-free. So let $\sigma_{i}\left(e_{0}\right)=1_{i}, e_{0} \in R$, and choose integers $s>r>0$ such that $e_{0}^{s}=e_{0}^{r}$. Let

$$
e=e_{0}^{(0-r) r} \text {. }
$$

Then $e$ is idempotent and, moreover, $\sigma_{i}(e)=1_{i}^{(--r) r}=1_{i}$. Also, $e$ is central (Claim 1 ), and hence $e$ is a nonzero central idempotent element of $R$. Thus, $e R$ is a ring with identity $e$. Because $e R$ inherits all the hypotheses of the ground ring $R$ (including $n$ torsion-free property), it follows by the first part of the proof that $e R$ is commutative, and hence

$$
[e x, e y]=0 \text { for all } x, y \in R .
$$

This implies (since $\sigma_{i}(e)=1_{i}$ )

$$
\left[\sigma_{i}(x), \sigma_{i}(y)\right]=0 \text { for all } x, y \in R,
$$


and thus $R_{i}=\sigma_{i}(R)$ is again commutative. Hence the ground ring $R$ is commutative, and the theorem is proved.

We conclude by giving examples which show that all the hypotheses of Theorems 1 and 2 are essential.

EXAMPLE 1: Let

$$
R=\left\{\left(\begin{array}{ccc}
a & b & c \\
0 & a^{2} & 0 \\
0 & 0 & a
\end{array}\right) \mid a, b, c \in G F(4)\right\},
$$

and let $n=6$. Then $R$ satisfies all the hypotheses of Theorem 2 except that $R$ is not $n$-torsion-free. Note that $R$ is not commutative, and hence the hypothesis " $R$ is n-torsion-free" cannot be omitted in Theorem 2.

EXMAPLE 2: Let $R$ be as in Example 1, and let $n=7$. Then $R$ satisfies all the hypotheses of Theorem 2 except the hypothesis " $(x y)^{n}-(y x)^{n} \in Z$ ", and hence this hypothesis cannot be omitted in Theorem 2.

EXAMPLE 3: Let

$$
R=\left\{\left(\begin{array}{lll}
a & b & c \\
0 & a & d \\
0 & 0 & a
\end{array}\right) \mid a, b, c, d \in G F(3)\right\},
$$

and let $n=7$. Then $R$ satisfies all the hypotheses of Theorem 2 except the hypothesis " $N$ is commutative", and hence this hypothesis cannot be omitted in Theorem 2 (note that $R$ is not commutative).

EXAMPLE 4: Let

$$
R=\left\{\left(\begin{array}{lll}
a & b & c \\
0 & a & d \\
0 & 0 & a
\end{array}\right) \mid a, b, c, d \in G F(2)\right\},
$$

and let $n=2$. This ring shows that the condition " $n$-torsion-free" cannot be omitted in Theorem 1.

EXAMPLE 5: Let $R$ be as in Example 4 but with entries in $G F(3)$, and let $n=2$. This ring shows that the condition " $\left[x^{n}, y^{n}\right]=0$ " cannot be omitted in Theorem 1 .

EXAMPLE 6: Let $R$ be as in Example 1 with $n=3$. This ring shows that the condition " $(x y)^{n+1}-x^{n+1} y^{n+1} \in Z$ " cannot be omitted in Theorem 1 .

EXAMPLE 7:

$$
R=\left\{\left(\begin{array}{lll}
0 & a & b \\
0 & 0 & c \\
0 & 0 & 0
\end{array}\right) \mid a, b, c \in G F(3)\right\},
$$

and let $n=4$. This ring shows that the condition " $1 \in R$ " cannot be omitted in Theorem 1. 


\section{REFERENCES}

[1] H. Abu-Khuzam, H. Tominaga, and A. Yaqub, 'Commutativity theorems for s-unital rings satisfying polynomial identities', Math. J. Okayama University 22 (1980), 111-114.

[2] H. Abu-Khuzam and A. Yaqub, 'n-torsion-free rings with commuting powers', Math. Japon. 25 (1980), 37-42.

[3] H. Abu-Khuzam, 'A commutativity theorem for periodic rings', Math. Japon. 32 (1987), 1-3.

[4] H. E. Bell, 'Some commutativity results for periodic rings', Acta Math. Acad. Sci. Hungar. 28 (1976), 279-283.

[5] H. E. Bell, 'On rings with commuting powers', Math. Japon. 24 (1979), 473-478.

[6] H. E. Bell, 'On commutativity of periodic rings and near-rings', Acta Math. Acad. Sci. Hungar. 36 (1980), 293-302.

[7] L. P. Belluce, I. N. Herstein, and S. K. Jain, 'Generalized commutative rings', Nagoya Math. J. 27 (1966), 1-5.

[8] I. N. Herstein, 'A commutativity theorem', J. Algebra 38 (1976), 112-118.

[9] S. Ikehata and H. Tominaga, 'A commutativity theorem', Math. Japon. 24 (1979), 29-30.

[10] W. K. Nicholson and A. Yaqub, 'A commutativity theorem for rings and groups', Canad. Math. Bull. 22 (1979), 419-423.

[11] W. K. Nicholson and A. Yaqub, 'A commutativity theorem', Algebra Universalis 10 (1980), 260-263.

Department of Mathematics

Kuwait University

Kuwait 13060

Department of Mathematics

University of California

Santa Barbara CA 93106

United States of America
Department of Mathematics

Brock University

St. Catharines

Ontario, Canada L2S $3 A 1$ 